\title{
Formation of cold filaments from colliding superbubbles
}

\author{
Evangelia Ntormousi ${ }^{1,2,3}$, Katharina Fierlinger ${ }^{1,4}$, Andreas Burkert ${ }^{1,2,4}$ \\ and Fabian Heitsch ${ }^{5}$ \\ ${ }^{1}$ University Observatory Munich, \\ Scheinerstr. 1, D-81679 München, Germany \\ email: eva.ntormousi@cea.fr \\ ${ }^{2}$ Max-Planck Institut für Extraterrestrische Physik \\ Giessenbachstrasse 185748 Garching \\ ${ }^{3}$ Service d'Astrophysique, CEA/DSM/IRFU \\ Orme des Merisiers, Bat 709 Gif-sur-Yvette, 91191 France \\ ${ }^{4}$ Excellence Cluster Universe \\ Boltzmannstr. 2 D-85748 Garching \\ ${ }^{5}$ Department of Physics and Astronomy \\ University of North Carolina Chapel Hill \\ Chapel Hill, NC 27599-3255
}

\begin{abstract}
We present results from numerical simulations of expanding and colliding supershells. These large-scale spherical shocks, created by the combined feedback from several OB stars, are unstable to a number of hydrodynamical instabilities, so they quickly fragment into cold and highly structured clumps. A collision between two large shells can organize these small clumps into very filamentary structures, of tens of parsecs length and less than a parsec thick. In simulations where the flow of stellar material is followed with a tracer quantity, cold structures practically do not contain any enriched material from the $\mathrm{OB}$ associations at the time of their creation. In this context then, the clumps are created almost exclusively out of diffuse ISM material, containing almost no wind or supernova matter. Although the mechanism presented here is possibly not the only route for filament creation, this predicted property may help identify regions of sequential star formation.
\end{abstract}

Keywords. ISM, hydrodynamics, stars:formation

\section{Introduction}

Massive stars deposit large amounts of energy and considerable amounts of mass to their surroundings during their lifetimes in the form of winds. Their deaths as supernova explosions are also a powerful source of energy and new metals for the Interstellar Medium (ISM).

It is well known by now that the collective feedback from several OB stars creates compressions in the ISM of scales that range from tens to hundreds of parsecs. These huge spherical shocks around OB associations are typically observed to fragment into molecular clouds and lead to new events of star formation; as a result the conditions for large-scale shock fragmentation have been the focus of extensive theoretical work. Although a full summary is not possible here, we refer the reader to the analysis by Vishniac (1983), who provided dispersion relations for the growth of dynamical instabilities on spherical shocks, Whitworth et al. (1994), who showed that the fragments become self-gravitating when the thin shock layer is still confined by ram pressure and Mac Low \& Norman 
(1993), who performed numerical studies of the Vishniac instability, as most relevant to the contents of this paper.

The purpose of this work is to explore the nonlinear behavior of the gas in large spherical thin interstellar shocks as the result of relevant hydrodynamical instabilities, namely as the Vishniac instability, ,the Kelvin-Helmholtz and the Thermal instability, by means of numerical simulations. We show that this interaction can create cold, dense structures in a variety of physical states and with supersonic internal velocities. The collision between two spherical shells creates very elongated cold filaments due to the large-scale shear.

The feedback from young OB associations is modeled as a time-dependent mass and energy source taken from stellar population synthesis models. In addition, the flow of the (expectedly metal-enriched) material from the hot to the cold phase is followed with a passive hydrodynamical quantity.

\section{Numerical Simulations}

High-resolution numerical simulations (maximum resolution of $0.1 \mathrm{pc}$ ) have been performed with the hydrodynamical Adaptive Mesh refinement (AMR) code RAMSES Teyssier(2002), in two and three dimensions. The time-dependent energy and mass input from OB associations, as calculated by Voss et al. (2009) is implemented in the code as a source term in the energy and in the mass equations following a reference table. In the same way, the heating and cooling rates typical of a solar metallicity gas are tabulated for various densities and temperatures and added or subtracted accordingly from the energy budget of each cell at every time step. In some of the simulations an additional continuity equation is solved for a tracer quantity, allowing us to follow the flow of wind and supernova material into the rest of the fluid.

\subsection{Filament formation}

All simulations start with diffuse, warm $\left(n_{H}=1 \mathrm{~cm}^{-3}, \mathrm{~T}=8000 \mathrm{~K}\right)$ gas, which corresponds to the warm thermally stable phase of the ISM as defined by the used cooling and heating rates. Two identical feedback regions representing OB associations of 20 or 50 stars each are placed on either side of the computational box. Thin dense shocks form rapidly around these regions. The simulations are allowed to continue well after the two shocks collide at the middle of the computational box and are stopped only when boundary effects start to become important. The typical morphology of a simulation is shown in Figure 1.

As the shocks decelerate through their interaction with the surrounding gas, the Vishniac instability magnifies any perturbations on the shock surface, resulting in characteristic triangular ripples. The shear inside the shock triggers the Kelvin-Helmholtz instability, while the condensations at the tips of the ripples are thermally unstable. The net result is the formation of small, cold and dense clumps. These clumps are at lower pressure than their environment, typical of the Thermal Instability, so many of them are observed to be condensing.

When the shells collide, the small clumps are brought together and at the same time sheared into a filamentary morphology. This is illustrated in the left panel of Figure 1. It is worth pointing out that it takes less than $1 \mathrm{Myr}$ for the clumps to form, a timescale much shorter that those typically estimated for the gravitational fragmentation of the shell alone. 

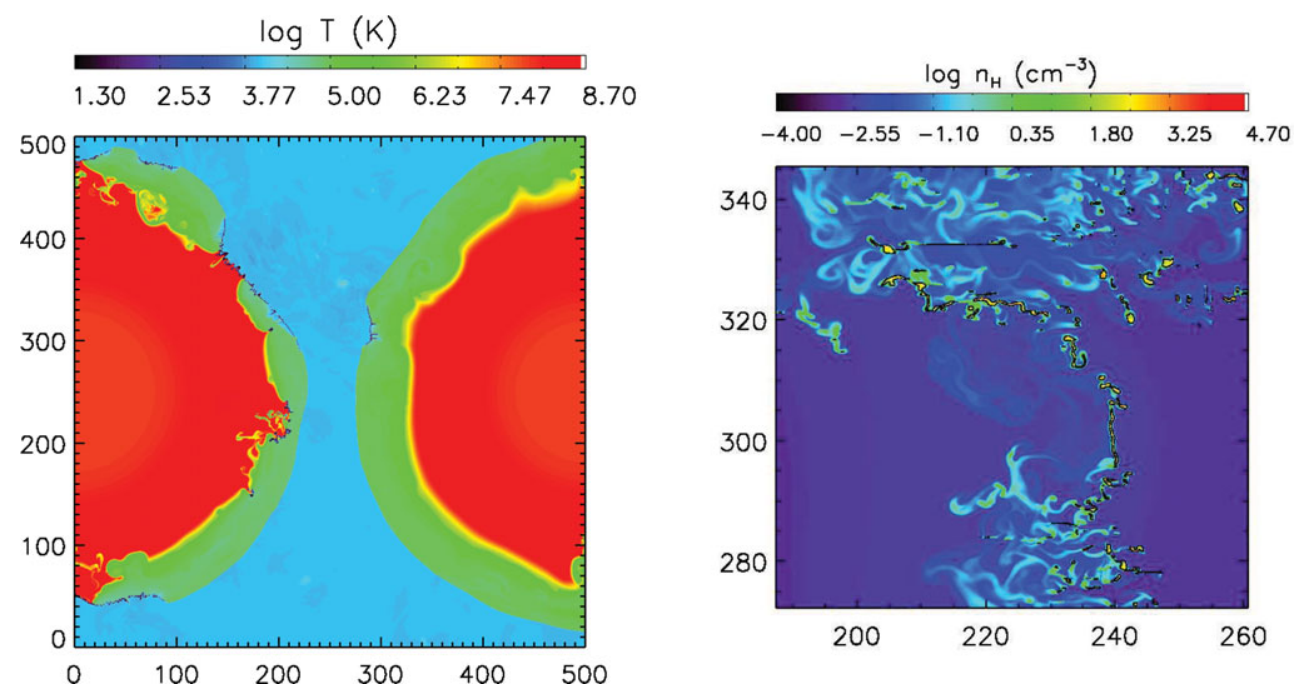

Figure 1. Left: Contour plot of the logarithm of the gas temperature at an early stage (about 3 Myrs after star formation started in the OB associations) of a 2D supershell collision simulation in a turbulent diffuse environment. Right: A zoomed-in region of a $2 \mathrm{D}$ supershell simulation at the shock collision interface, about 7 Myrs since star formation started in the associations. Shown here are contours of the logarithm of the hydrogen number density. The contour for $n_{H}=$ $50 \mathrm{~cm}^{-3}$ is shown in black. The small-scale clumps are grouped in a filamentary configuration due to the large-scale shear. The axes are in parsecs. These Figures can also be found in Ntormousi et al. (2011).

\subsection{Flow of material from the hot to the cold gas}

The flow of material from the hot and metal-enriched gas to the cold clumps formed around the associations is traced in the simulations by adding an arbitrary amount of a tracer quantity in the feedback regions at each timestep. The behavior of the flow is illustrated by the plots in Figure 2. It is evident that the clumps are formed out of the warm diffuse phase of the ISM compressed by the shocks. Surprisingly, the hot gas is not mixed into them even at late times, when the shells collide and the clumps are found in the very turbulent interaction region. Since accretion due to gravity has not been modeled, we cannot draw strong conclusions for the future metallicity evolution of the clumps, but what is evident from these simulations is that at the time of their formation the clumps are composed purely out of the pre-existing diffuse gas.

\section{Conclusions}

A series of numerical simulations exploring the nonlinear evolution of the gas inside and around these large-scale spherical shocks show that cold filamentary structures of a few parsecs size form naturally and rapidly around OB associations. Collisions between such shocks create much longer filaments, of the order of tens of parsecs.

In those simulations where we can trace the flow of hot gas we observe that the cold clumps are formed at the outer part of the shock and thus contain no wind or supernova material from the stars that triggered their existence. The turbulence created at the collision interface does not help mixing into the coldest gas, although it does help mixing between the hot and the warm gas. In order to estimate the final metallicities of the clumps further investigation is needed, including modeling of thermal conductivity to 

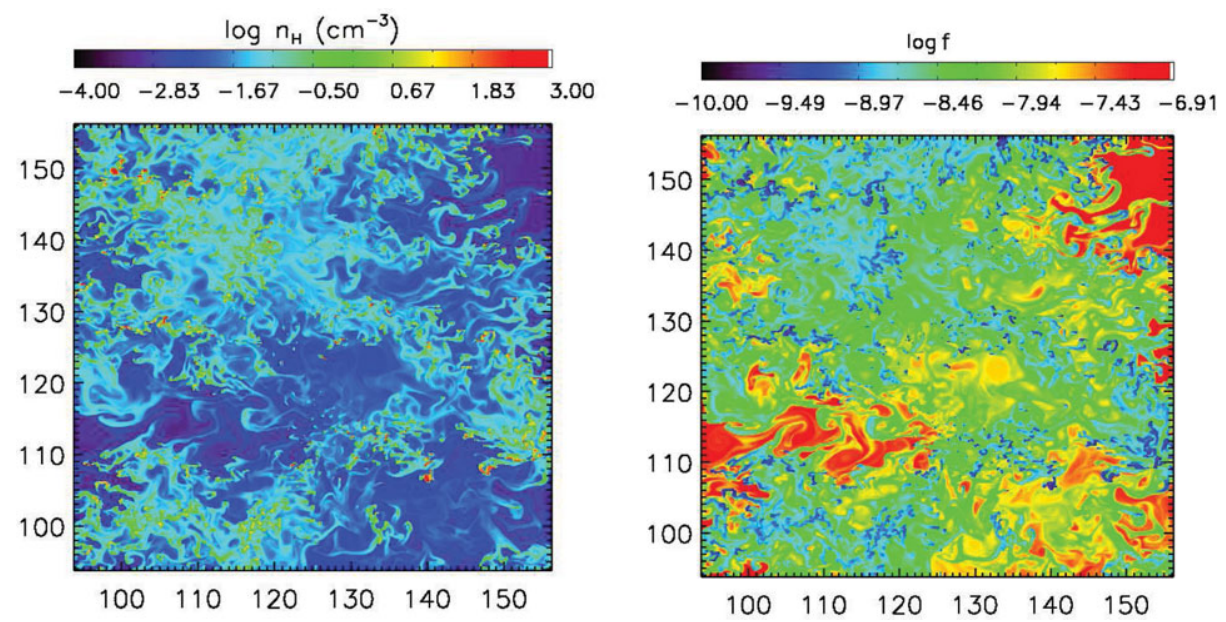

Figure 2. Contour plots of the logarithm of hydrogen number density (left) and the logarithm of relative tracer content (right) at the shock collision interface in a $2 \mathrm{D}$ simulation, about 3 Myrs since star formation started. The axes are in parsecs, like above, although this simulation was done in a smaller domain. It is clear that dense regions contain practically no new material. This Figure can also be found in Ntormousi \& Burkert (2011).

study clump evaporation, possible inhomogeneities in the metal input and gravitational accretion from the environment.

\section{References}

Mac Low, M.-M. \& Norman, M. L. 1993, ApJ, 407, 207

Ntormousi, E. \& Burkert, A. 2011, arXiv:1111.1859

Ntormousi, E., Burkert, A., Fierlinger, K., \& Heitsch, F. 2011, ApJ, 731, 13

Teyssier, R. 2002, A\&A, 385, 337

Vishniac, E. T. 1983, ApJ, 274, 152

Voss, R., Diehl, R., Hartmann, D. H., et al. 2009, A\&A A, 504, 531

Whitworth, A. P., Bhattal, A. S., Chapman, S. J., Disney, M. J., \& Turner, J. A. 1994, A\&\&A, 290,421

\section{Discussion}

WANG: Do you include thermal conduction in your simulations?

NTORMousi: Unfortunately the presence of very hot gas in these simulations would make the timesteps extremely small if thermal conduction was included. However, we are currently resimulating smaller regions of the larger volume at higher resolution, including thermal conductivity. Qualitatively, we expect the effect of thermal conduction to be cloud evaporation. This would essentially dilute the metal content of the warm gas rather than lead to any enrichment of the dense gas. We will report detailed results after the new simulations are completed.

Cesarsky: What do these clouds become? Are the filaments you see in your simulations related to the filaments observed by Philippe Andre and others with Herschel in star forming and non-star forming interstellar clouds? 
NTORMOUsi: In our simulations we do not yet include chemistry, so we cannot directly compare our results with observations. Certainly the filaments formed in these numerical experiments bear strong resemblance to dense filamentary interstellar clouds and have very dynamical nature. I could imagine the Pipe nebula, for example, to have formed in this way, since it is located near an OB association, the same for the Lupus-Ophiuchus cloud complexes, which are between two OB associations. 